\title{
Status of NNLO QCD corrections for process with one or more jets in the final state at the LHC
}

\section{João Pires $^{a, b, *}$}

${ }^{a}$ LIP, Avenida Professor Gama Pinto 2, P-1649-003, Lisbon, Portugal

${ }^{b}$ Faculdade de Ciências, Universidade de Lisboa, 1749-016 Lisboa

E-mail: jnpires@lip.pt

The abundant amount of data to be collected by the ATLAS and CMS collaborations in future runs of the Large Hadron Collider at CERN opens up a new era of precision physics. Some of the most prominent precision observables are related to processes with one or more jets in the final state. In order to fully exploit the potential of the LHC and the HL-LHC, it is imperative to make theoretical predictions at the level of accuracy that matches or even exceeds that of the upcoming measurements. In this talk we present a review of the status of theoretical predictions including NNLO QCD corrections for process with one or more jets in the final state at the LHC.

*** Particles and Nuclei International Conference - PANIC2021 ***

*** 5 - 10 September, $2021 * * *$

*** Online ***

${ }^{*}$ Speaker 

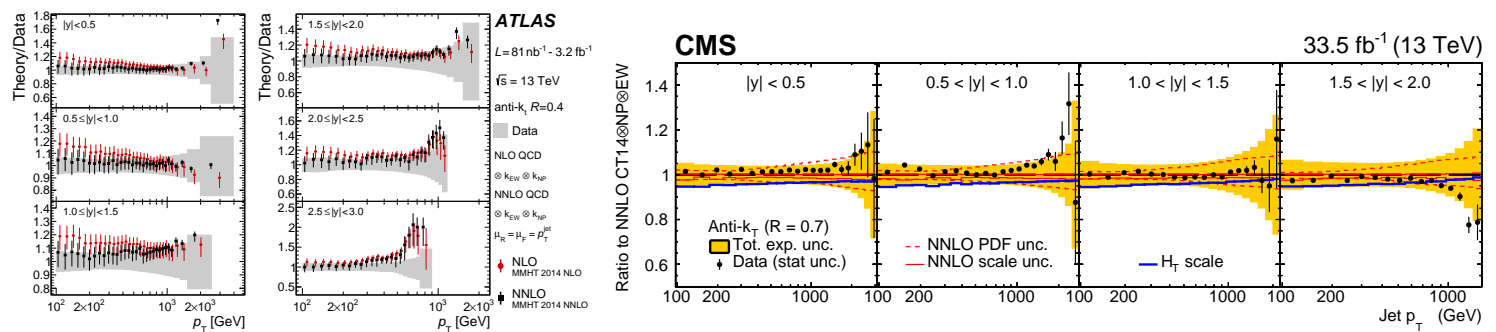

Figure 1: Ratios of NNLO pQCD predictions and $\sqrt{s}=13 \mathrm{TeV}$ LHC measurements from ATLAS [5] (left) and CMS [6] (right) for inclusive jet production.

\section{Introduction}

The Large Hadron Collider (LHC) is currently colliding protons at centre of mass energies up to $\sqrt{s}=13 \mathrm{TeV}$, with the goal of searching the high energy frontier for signs of physics beyond the Standard Model. In this presentation we review the status of the NNLO QCD corrections for processes with one or more jets in the final state at the LHC.

\section{Inclusive jet production}

The perturbative calculations for the inclusive jet and dijet cross sections at NNLO in QCD have recently been completed by two groups [1-4]. In Fig. 1 we show comparisons between fixed-order NLO and NNLO QCD predictions to ATLAS (left) and CMS (right) $13 \mathrm{TeV}$ inclusive jet- $p_{T}$ spectrum data for anti- $k_{T} R=0.4$ (ATLAS) and $R=0.7$ (CMS) cone sizes, corrected for non-perturbative and electroweak effects. We observe a significant improvement in the description of the jet data when going from NLO to NNLO and a smaller impact of the higher order corrections for the $R=0.7$ jet cone size.

A calculation of dijet production at NNLO in QCD has been presented in [3]. In Fig. 2 we present a comparison of the theory prediction at fixed order corrected for non-perturbative and electroweak effects, to the CMS dijet $8 \mathrm{TeV}$ triple differential dataset. We observe that the NNLO prediction changes both the shape and normalization of the NLO result and has significantly
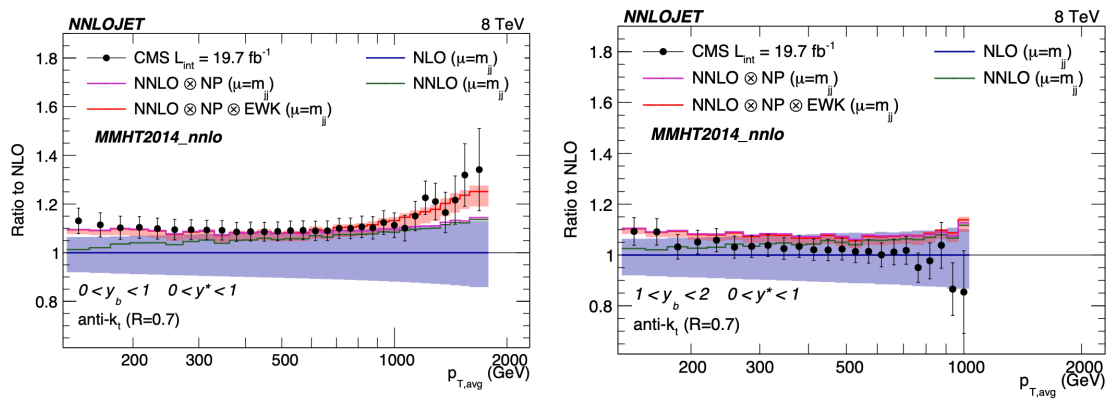

Figure 2: Ratios of NLO and and NNLO theory predictions and CMS data normalized to the NLO central value. The shaded bands shown for the NLO (blue) and the NNLO corrected for non-perturbative and electroweak effects (red) represent the theory uncertainty from variation of the renormalization and factorization scales in the calculation [3]. 

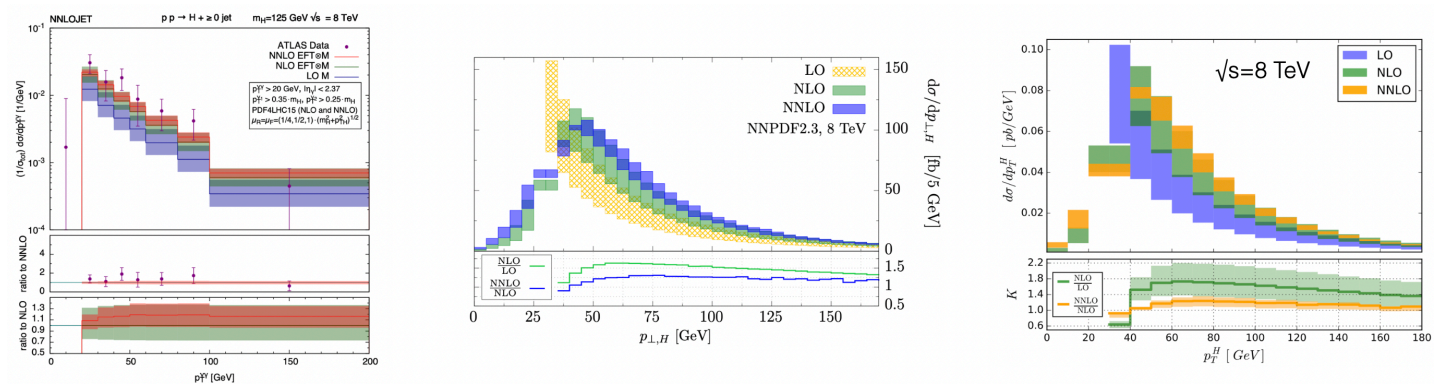

Figure 3: Higgs boson transverse momentum distribution calculations at LO, NLO and NNLO in the publications [7] (left) [8] (middle), [9] (right) and ATLAS data from ref [11]. Shaded bands in the plots represent the theoretical scale uncertainty in the calculations.

reduced theory uncertainties. Overall the agreement with the data is excellent at small $y_{b}$, but for events with large dijet boost kinematics, the data sits below the theory prediction. In this region, which is sensitive to the scattering of large- $x$ parton on a low- $x$ parton, the PDFs suffer from large uncertainties.

\section{Higgs boson plus jet production}

The determination of the Higgs boson properties is a central goal at the LHC and HL-LHC, where it is expected that the predictions of the SM will be tested to the five percent level in several production and decay modes. For this reason, to improve the modelling of the Higgs kinematics at the LHC, precise predictions for Higgs production in association with jets are needed. The number of jets produced in association with a Higgs boson candidate is a very important discriminator between different production modes, and plays a key role in the background rejection for many Higgs boson studies.

Fixed-order predictions for $H+$ jet at NNLO in QCD have been calculated in [7-9], in the effective theory approximation, obtained by integrating out the top quark. The full top-quark mass dependence for $H+$ jet is currently known at NLO only [10]. In Fig. 3 we show results for the Higgs $p_{T}$-spectrum at NNLO. We observe large radiative corrections and a good agreement between the NNLO prediction reweighted by exact top-mass dependence at LO and the ATLAS ( $H \rightarrow \gamma \gamma$ ) data normalized to the total inclusive Higgs cross section.
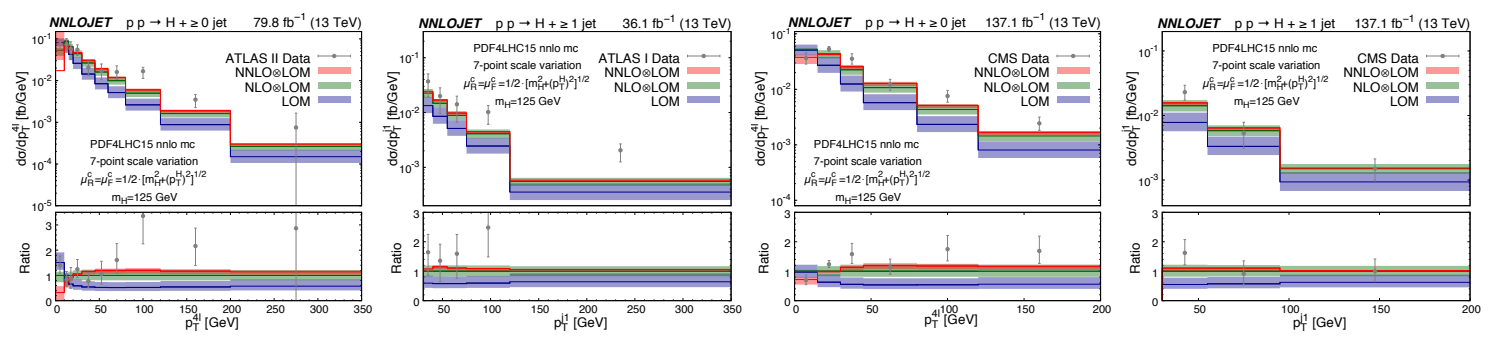

Figure 4: Transverse momentum distributions of the Higgs boson and of the leading jet produced in association with a Higgs boson at LO, NLO and NNLO [12] compared with ATLAS [13, 14] and CMS [15] data. 
The four-lepton decay mode of the Higgs boson allows for a clean kinematic reconstruction, thereby enabling precision studies of the Higgs boson properties and of its production dynamics. In Fig. 4 we show the NNLO QCD corrections to fiducial cross sections relevant to this decay mode in the gluon-fusion production of a Higgs boson in association with a hadronic jet. In this calculation [12] we can take into account fiducial cuts on the Higgs boson decay products as well as on accompanying objects such as hadronic jets. We observe that the NNLO corrections are sizeable and kinematics dependent. With respect to NLO we observe a substantial reduction of scale uncertainties of the theory prediction to a level of about $10 \%$ in most distributions [12].

\section{Beyond $2 \rightarrow 2$ processes}

In this section we present recent results on the calculation of NNLO jet cross sections for processes beyond $2 \rightarrow 2$ scattering. These processes are the current frontier of research, for which the required two-loop 5-point amplitudes have been only recently derived. In particular, two loop amplitudes for diphoton+jet production and 3-jet production have been derived in [16] and [17, 18].

The $\gamma \gamma+$ jet NNLO calculation presented in [19] represents the first NNLO-accurate prediction for the transverse momentum distribution of the diphoton system. This observable represents the main background for Higgs production at high- $p_{T}$, and is relevant for dedicated measurements of diphoton production. The results show that NNLO is the first order where the perturbative expansion converges with overlapping scale uncertainty bands between two consecutive orders. The scale uncertainty in the observable is at the 1 2\% level across the most part of the distribution.

In Fig.5 we present distributions for three jet production at NNLO in QCD calculated in [20]. This process has been studied in great detail by the experimental collaborations with the aim of providing a unique testing of perturbative $\mathrm{QCD}$, by comparing theory predictions with collider jet data.

In Fig.5 (left) we can observe that the NNLO corrections are of the of the order of $-15 \%$ at low- $p_{T}$ and increase steadily at high- $p_{T}$ for the first and second jet- $p_{T}$ spectrum. The 3rd jet- $p_{T}$ distribution is well-behaved: it has a flat NNLO corrections and fairly symmetric uncertainty bands at both NLO and NNLO. In the same figure on the right it is shown the three-jet to two-jet ratio as
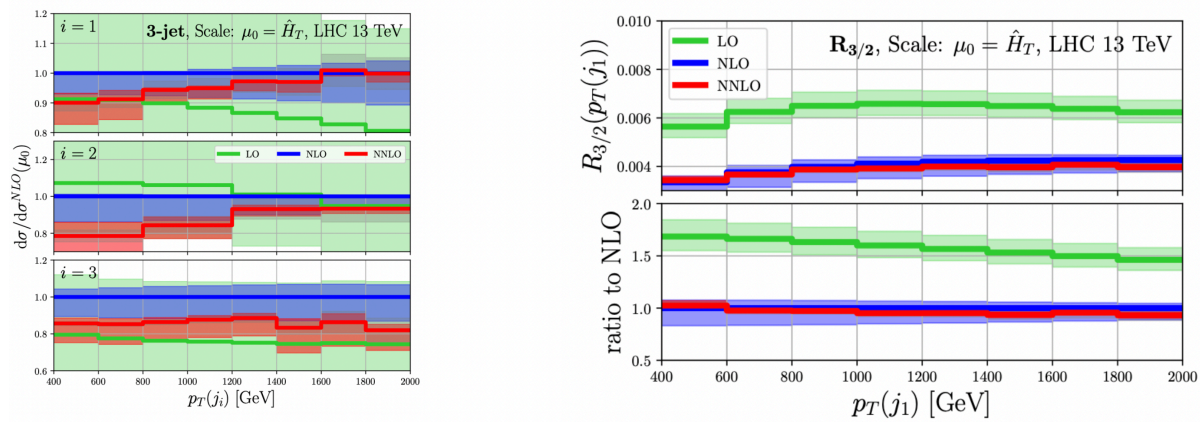

Figure 5: Transverse momentum distribution of the three leading jets for three jet production at LO, NLO and NNLO (left). The three jet to two jet ratio as a function of the leading jet $p_{T}$ (right). The colored bands in the plots represent the theoretical scale uncertainty in the calculations [20]. 
a function of the $p_{T}$ of the leading jet. We can observe that the prediction for the ratio is stabilised at NNLO with scale uncertainties at the $3 \%$ level.

\section{Conclusions}

Calculations of NNLO QCD corrections for many important $2 \rightarrow 2$ and $2 \rightarrow 3$ processes with jets in the final state at the LHC are now available and were reviewed in this presentation. The impressive progress in this field has been made possible due to the development of subtraction methods to treat infrared divergences at this order, and due to many advances in the calculation of multi-loop integrals and amplitudes. Given the amount of experimental data due to be collected during the LHC and HL-LHC runs, we anticipate that these results will allow us to perform physics analyses with LHC data at a new level of precision.

\section{References}

[1] Phys. Rev. Lett. 118 (2017) no.7, 072002 [arXiv:1611.01460 [hep-ph]].

[2] Phys. Rev. Lett. 119 (2017) no.15, 152001 [arXiv:1705.10271 [hep-ph]].

[3] Phys. Rev. Lett. 123 (2019) no.10, 102001 [arXiv:1905.09047 [hep-ph]].

[4] JHEP 10 (2019), 262 [arXiv:1907.12911 [hep-ph]].

[5] M. Aaboud et al. [ATLAS], JHEP 05 (2018), 195 [arXiv:1711.02692 [hep-ex]].

[6] A. Tumasyan et al. [CMS], [arXiv:2111.10431 [hep-ex]].

[7] JHEP 10 (2016), 066 [arXiv:1607.08817 [hep-ph]].

[8] Phys. Rev. Lett. 115 (2015) no.8, 082003 [arXiv:1504.07922 [hep-ph]].

[9] Phys. Lett. B 748 (2015), 5-8 [arXiv:1505.03893 [hep-ph]].

[10] Phys. Rev. Lett. 120 (2018) no.16, 162001 [arXiv:1802.00349 [hep-ph]].

[11] G. Aad et al. [ATLAS], JHEP 09 (2014), 112 [arXiv:1407.4222 [hep-ex]].

[12] JHEP 07 (2019), 052 [arXiv:1905.13738 [hep-ph]].

[13] M. Aaboud et al. [ATLAS], JHEP 10 (2017), 132 [arXiv:1708.02810 [hep-ex]].

[14] G. Aad et al. [ATLAS], Eur. Phys. J. C 80 (2020) no.10, 942 [arXiv:2004.03969 [hep-ex]].

[15] A. M. Sirunyan et al. [CMS], Eur. Phys. J. C 81 (2021) no.6, 488 [arXiv:2103.04956 [hep-ex]].

[16] JHEP 07 (2021), 164 [arXiv:2103.04319 [hep-ph]].

[17] JHEP 20 (2020), 167 [arXiv:2009.07803 [hep-ph]].

[18] JHEP 07 (2021), 095 [arXiv:2102.13609 [hep-ph]]. 
[19] JHEP 09 (2021), 093 [arXiv:2105.06940 [hep-ph]].

[20] Phys. Rev. Lett. 127 (2021) no.15, 152001 [arXiv:2106.05331 [hep-ph]]. 